G. FISCHER, G. MANN, M. PANCHENKO, and P. ZARKA (Eds.)

PLANETARY RADIO EMISSIONS VIII 


\section{Editorial team:}

Georg Fischer

Space Research Institute

Austrian Academy of Sciences

Graz, Austria

Gottfried Mann

Leibniz-Institut für Astrophysik

Potsdam, Germany

Mykhaylo Panchenko

Space Research Institute

Austrian Academy of Sciences

Graz, Austria

Philippe Zarka

Observatoire de Paris

Meudon, France

Webpage design

and editorial help:

Manuel Scherf

\section{Proofreading:}

Mike Kaiser

\section{Local Organizing Committee:}

Georg Fischer

Claudia Grill

Doris Hradecky

Maxim Khodachenko

Mykhaylo Panchenko

Manuel Scherf 
G. FISCHER, G. MANN, M. PANCHENKO, and P. ZARKA (Eds.)

\section{PLANETARY RADIO EMISSIONS VIII}

Proceedings of the 8Th International Workshop ON Planetary, Solar and Heliospheric Radio Emissions

held at Seggauberg near Graz, Austria

OCTOBER $25-27,2016$ 
Angenommen durch die Publikationskommission der mathematischnaturwissenschaftlichen Klasse der österreichischen Akademie der Wissenschaften: Friedrich G. Barth, Georg Brasseur, Karlheinz Erb, Josef Smolen, Christoph Spötl, Michael Wagner, Anton Zeilinger

Diese Publikation wurde einem anonymen, internationalen Peer-Review-Verfahren unterzogen.

This publication has undergone the process of anonymous, international peer review.

Die verwendeten Papiersorten sind aus chlorfrei gebleichtem Zellstoff hergestellt, frei von säurebildenden Bestandteilen und alterungsbeständig.

Alle Rechte vorbehalten.

ISBN 978-3-7001-8263-4

Copyright (C) 2017 by

Österreichische Akademie der Wissenschaften, Wien

Druck und Bindung: Ferdinand Berger \& Söhne GmbH, A-3580 Horn

Printed and bound in the EU

https://epub.oeaw.ac.at/8263-4

https://verlag.oeaw.ac.at 